\title{
CHOLECALCIFEROL ATTENUATES INDUCED PARKINSON'S LIKE-DISEASE VARIATION AND CELLULAR MORPHOLOGY OF STRIATUM AND SUBSTANTIAL NIGRA
}

\author{
Adekeye A.0 ${ }^{1,2}$, Adefule A.K ${ }^{2}$, Shallie P2,4 , Akpan H.B ${ }^{2}$, Adekomi D.A ${ }^{3,4}$ \\ 1. Department of Anatomy, College of Medicine and Health Sciences, Afe Babalola University, \\ Ado Ekiti, Nigeria \\ 2. Department of Anatomy, Faculty of Basic Medical Sciences, Olabisi Onabanjo University, \\ Ago-Iwoye, Nigeria. \\ 3. Department of Anatomy, Osun State University, Osogbo, Nigeria \\ 4. Department of Clinical Anatomy, Nelson R. Mandela School of Medicine, University of \\ Kwazulu-Nata, Durban, South Africa
}

Correspondence address: Adekeye A.O, Department of Anatomy, College of Medicine, Afe Babalola University; Email:, Tel: +2348038571625.

\begin{abstract}
Parkinson's disease is the commonest motor neurodegenerative disorder which affects the dopaminergic neurons and causes significant loss of dopamine. 1-methyl 4-phenyl-1,2,3,6tetrahydropyridine (MPTP) is a selective neurotoxin in the nigrostriatal pathway leading to this motor disorder. Cholecalciferol (Vitamin $\mathrm{D}_{3}$ ) has been described as an active neurosteriod with antioxidant properties ubiquitously present in the brain. The study hypothesized that stimulation of vitamin $D$ receptor by cholecalciferol could reduce autophagic cell death and degeneration following a state of drug induced parkinsonism in mice. The aim of the research was to observe the cytoarchitectural, histochemical, neurobehavioural and immunohistochemical effects of cholecalciferol on striatum and substantia nigra in mice model of MPTP-induced Parkinson's disease. Fifty adult male C57BL/6] mice weighing about $25-35 \mathrm{~g}$ were randomly selected and assigned into 5 groups for this study. The mice were then subjected to neurobehavioural, neurochemical and neuropathological evaluations. The results obtained showed a significant reduction $\left({ }^{*} p<0.05\right)$ in the estimated markers of oxidative stress with high dose of vitamin $D_{3}$ following MPTP induction. There was also statistical significant reduction $(* * p<0.01, * * * p<0.001)$ in the expression of GFAP-immuno-positive cells in the substantia nigra of the experimental mice when compared with the control group. It can be inferred that the administration of Vitamin $D_{3}$ was associated with significant attenuation of focal effects linked with MPTP in mice model of Parkinson's Disease.
\end{abstract}

Keywords: Aging, Neurodegeneration, Dopaminergic neuron, Vitamin $D_{3}$, Environmental toxins

\section{INTRODUCTION}

Parkinson's disease (PD) is the most common neurodegenerative movement disorder that affects approximately $1 \%$ of the population at 65 years of age and increases to $5 \%$ at 85 years (Van Den Eeden et al., 2003). The motor symptoms are collectively called Parkinsonism or Parkinsonian Syndrome (Shahed and
Jankovic 2007; Poewe, 2008). Reports have shown clinically that PD is characterized principally by four major symptoms such as resting tremor, bradykinesia, rigidity and postural instability (Lieberman, 2006). Pathologically, PD is characterized by the presence of proteinaceous inclusions called 
Lewy bodies and the biochemical hallmark is a profound deficit in brain dopamine due to the degeneration of dopaminergic neurons (DA) in the substantia nigra pars compacta (SNpc) of the midbrain leading to movement disorder which are poorly understood based on epidemiological survey in Sub-Saharan Africa using Nigeria as a case study and this may contribute to reduction in the quality of life of people especially the elderly (Okubadejo et al., 2010; Akinyemi, 2012). It is important to stress that Parkinson's disease is not restricted to the dopaminergic systems alone but involves widespread neuronal loss that can be detected in other catecholaminergic and noncatecholaminergic nuclei (Braak and Braak, 2000). Environmental risk factors for PD have received significant interest especially in Nigeria (Akinyemi, 2012) but the importance of genetic factors underlying the likelihood of developing PD is increasingly recognized (Jason et al., 2005). Although most cases of PD are sporadic, specific mutations in some genes cause familial form of PD which has led to a new approach in the study of PD (Wang et al., 2001, 2006). The identification of several mutant genes causing early-onset PD such as a-synuclein, UCHL1 (ubiquitin carboxyterminal hydrolase L1), parkin, DJ1 and PINK1 (putative serine threonine kinase) has yielded crucial insights into the possible pathogenic mechanisms (Dawson et al., 2003; Dawson et al., 2010; Bras et al., 2015). Oxidative stress contributes to the cascade events leading to dopamine cells degeneration in Parkinson's disease (Jenner, 2003; Chiu et al., 2012). A cell is said to be in a state of oxidative stress when the amount of reactive oxygen species (ROS) exceeds a certain threshold and the cell no longer functions effectively, leading to cell death (Wyrsch et al., 2012). However, oxidative stress is intimately linked to other components of the degenerative processes such as mitochondrial dysfunction, excitotoxicity, nitric oxide toxicity and inflammation (Jenner, 2003; Chiu et al., 2012). Recently, altered ubiquitination and degradation of proteins have been implicated in dopaminergic cell death in Parkinson's disease. Mitochondria dysfunction has also been linked to inhibition of the electron transport chain and this inhibition may lead to generation of ROS and depletion of cellular energy level, which can consequently cause cellular damage or death mediated by oxidative stress and excitotoxicity (Dauer et al., 2002; Song et al., 2004). Mutations to mitochondria can occur as a result of exposure to environmental toxins such as pesticides (maneb, rotenones) or they can occur as a result of genetics and this may directly affect cell's ability to secrete dopamine (Ciccheti et al., 2005). A recent study postulated that low levels of Vitamin D during early life may be relevant to several brain diseases such as schizophrenia and multiple sclerosis (McGrath, 2001; James and Asuni, 2013). Previous studies have confirmed that VDR is expressed in both developing and adult rat brain as well as in adult hamster brain (Burkert et al., 2003; James and Asuni, 2013). Vit. $D_{3}$ may be synthesized locally within the brain via expression of the enzyme 1a- hydroxylase (1aOHase) which catalyses conversion of $25 \mathrm{OHD}_{3}$ to $1,25(\mathrm{OH})_{2} \mathrm{D}_{3}$. Both the receptor and the enzyme (1a-OHase) required for the production of the active form of vitamin $D$ were identified in neuronal and glial cells (Darryl et al., 2004; Groves et al., 2014). Studies have shown that dopamine is able to induce VDRmediated signalling in the absence of the ligand suggesting a complex interaction between Vitamin $\mathrm{D}$ and neurotransmitters (Dulla et al., 2016; Uberti et al., 2016). Vitamin $\mathrm{D}_{3}$ also play an important role in the pathogenesis of skeletal disorders and calcium homeostasis (Groves et al., 2014). MPTP toxicity is due to inhibition of complex- 1 of the mitochondrial electron transport chain, which leads to lack of ATP and causes cell death. In $\mathrm{PD}$, there is a $30 \pm 40 \%$ decrease in complex-1 activity in the substantia nigra pars compacta which likely adds to energy failure and also predisposes the pars compacta to toxic substances and increases its proneness to cell death (Kingsbury et al., 2001; Chung et al., 2010; Kim et al., 2016; Brugnoli et al., 2016). 


\section{MATERIALS AND METHODS}

\section{Animals and treatment}

50 Male adult C57BL/6Jw mice weighing 25$35 \mathrm{~g}$ were procured from Afe Babalola University Animal holding and used in the study. They were left to acclimatize for two weeks and were later randomly divided into 5 groups (A-E) with light and dark cycle controlled. Food and water were made available ad libitum except during the experiments. All experimental procedures were carried out in accordance with Ethical Committee of the Olabisi Onabanjo University, Nigeria and also in line with the National Institute of Health Guide for care and Use of Laboratory Animals (NIH Publication, 2011) as stated in the "Guide to the care and use of Laboratory Animal Resources. Efforts were made to keep the number of animals used to the lowest possible and to minimise animal suffering. Group A $(n=10)$ received normal saline for 28 days, representing the control group (NS). Group B $(n=10)$ was given MPTP at a dose of $20 \mathrm{mg} / \mathrm{kg}$ BW intraperitoneally for 14 days to induce $\mathrm{PD}$, following which they were given Vitamin $D_{3}$ (IP; $50 \mathrm{mg} / \mathrm{kg} \mathrm{BW}$ ) for another 14 days as a post treatment of MPTP (MPTP/Vit. $\left.\mathrm{D}_{3} \mathrm{~L}\right)$. Group C $(n=10)$ were also administered MPTP intraperitoneally for 14 days at a dose of $20 \mathrm{mg} / \mathrm{kg}$ BW, following which they were treated with Vitamin $\mathrm{D}_{3}$ (IP; $100 \mathrm{mg} / \mathrm{kg}$ BW) for 14 days (MPTP/Vit. $\mathrm{D}_{3}{ }^{\mathrm{H}}$ ). Group D $(n=10)$ received MPTP intraperitoneally at a concentration of $20 \mathrm{mg} / \mathrm{kg}$ BW for 14 days after which they were given LDOPA (IP; $5 \mathrm{mg} / \mathrm{kg} \mathrm{BW}$ ) for 14 days as post treatment with MPTP (MPTP/L-DOPA). Group E $(n=10)$ received normal saline for 14 days after which MPTP intraperitoneally at a concentration of $20 \mathrm{mg} / \mathrm{kg}$ BW for 14 days was administered (MPTP only). Cholecalciferol (Vit.D ${ }_{3}$, L-DOPA, MPTP, antibodies and all consumables were purchased from SigmaAldrich, Canada and Medchem Express, USA. The water used was glass-distilled and other reagents were of analytical grade.

\section{Neurobehavioural studies}

Motor activity and coordination was measured in the animals using different battery of tests such as open field, rotarod and parallel bar tests. These tests were carried out in a closed room with proper illumination and control in behavioural room. At the end of the treatments, all tests were done on mice using a digital video recorder and were analysed later for motor activity and coordination.

\section{Animal sacrifice}

Mice were sacrificed $24 \mathrm{~h}$ after the last injection and neurobehavioural studies, based on previous studies that revealed a significant activation of astroglia (Frau et al., 2012). Five (5) mice were sacrificed through cervical dislocation and transcardially perfused with paraformaldehyde (4\% in $0.1 \mathrm{M}$ phosphate buffer, pH 7.4). The animals were then decapitated, skull exposed and brains excised out. The brains were then kept in a specimen bottle containing $10 \%$ formal saline for further processing (Histopathology and immunohistochemistry). Another 5 mice's brains from each group were kept in a specimen bottle containing $30 \%$ sucrose in a frozen environment $\left(4^{\circ} \mathrm{C}\right)$ in order to carry out biochemical analysis for markers of oxidative stress. For immunohistochemistry studies, sections from the CPu and SNc (7 $\mu$ m thick) were coronally cut on a microtome and immunoreacted with primary antibodies directed against GFAP (monoclonal mouse anti-GFAP, 1:400, Sigma, Italy). For diaminobenzidine (DAB) visualization of GFAP, the proper biotinylated secondary antibody (goat anti-mouse IgG for GFAP from Vector, United Kingdom) was used and the avidinbiotin-peroxidase protocol (ABC, Vector, United Kingdom) was applied in accordance with Frau et al., (2012). Sections were mounted on gelatin-coated slides, dehydrated and cover slipped before the analysis. Analysis of GFAP immunoreactivity images were digitized under constant light conditions with a camera using a software called image $\mathrm{J}$ and captured at $400 \mathrm{x}$ magnification. For each mouse, three sections 
$(A=1.10 \mathrm{~mm} ; 0.74 \mathrm{~mm} ; 0.38 \mathrm{~mm}$ from bregma) according to Paxinos and Franklin (2008) were captured, then one portion from the dorsolateral $\mathrm{CPu}$ and one portion from the ventromedial $\mathrm{CPu}$, left and right, were analysed in each section. The number of GFAP-positive cells in each section was manually counted and the single value obtained for each striatal level analysed was first normalized with respect to correspondent control group then values from different levels were averaged.

\section{Statistical analysis}

Data were presented as mean $\pm \mathrm{SEM}$; analysed using one-way ANOVA and Turkeys post hoc test. Statistical Significance was set as $\mathrm{P}<0.05^{*}$. Graphs were drawn with the aid of GraphPad Prism software.

\section{RESULTS}

\section{Motor function test}

Motor coordination was assessed in the experimental animals using open field test, rotarod and parallel bar test.

\section{Open Field Test}

Locomotor activity was measured in the experimental animals using open field test for 5 minutes. The animals were exposed to a large square box and allowed to move freely. The number of lines crossed plus number of rearing which is the assessment of locomotive behaviour was recorded and plotted in a graph (Figure 1). Statistically significant difference was seen when the number of line crossed within the treatment groups was analysed and compared with the control group using oneway ANOVA $\left({ }^{*} p<0.05\right)$. MPTP/Vit. $\mathrm{D}_{3}{ }^{\mathrm{H}}$ group seems to be more significant when compared with normal saline and MPTP only group which implies that total locomotor activity was significantly affected by vitamin $\mathrm{D}_{3}$ at high dose $(* * * p<0.001)$. MPTP/Vit. $D_{3} L$ was slightly significant in terms of total locomotor activity when compared with the control group and MPTP group only $\left({ }^{*} P<0.05\right)$. The figure 1 below showed bar chart representing the total locomotive activities in the open field test among experimental animals. MPTP/Vit. $\mathrm{D}_{3}{ }^{\mathrm{H}}$ group seems to be more significant when compared with normal control group and MPTP only group $(* * * p<0.001)$. Vitamin $D_{3}$ at high dose (MPTP/Vit. $D_{3} L$ ) was slightly significant when compared with the control group and MPTP group only ( $\left.{ }^{*} \mathrm{P}<0.05\right)$.

\section{Rotarod Test}

Motor coordination of the experimental animals was measured using rotarod test for 5 minutes. Animals were placed on rotating bar of the rotarod and the time it takes the animals to fall off the rotating bar or cling to side wall of the machine was recorded (Latency of Fall - LOF). Animals with good motor coordination are expected to spend more time on the rotating bar. Group C animals (treated with $20 \mathrm{mg} / \mathrm{kg}$ MPTP and $100 \mathrm{mg} / \mathrm{kg}$ Vit. $D_{3}$ ) had an increase in LOF value (Figure 2), which is statistically significant when compared to the control group (NS) and MPTP group only $\left({ }^{*} p<0.05\right.$, $* * p<0.01)$. 


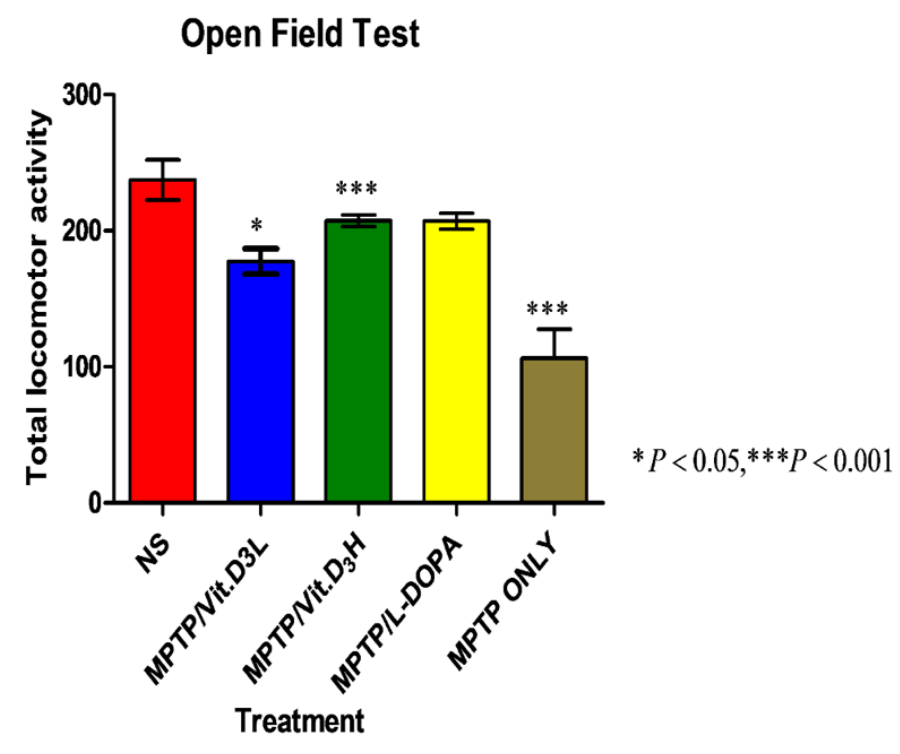

Figure 1: Bar Chart showing TLA by the experimental animals in the OFT
Latency of fall in the Rotarod test carried out on the experimental animals (Figure 2). The Bar chart showed that there was a statistical significant difference in the latency of fall (LOF) in MPTP/Vit. $\mathrm{D}_{3}{ }^{\mathrm{H}}$ (treated with $20 \mathrm{mg} / \mathrm{kg}$ MPTP and $100 \mathrm{mg} / \mathrm{kg}$ Vit. $D_{3}$ ) when compared with MPTP treated group only $(* p<0.05)$ but no statistical significant (ns) difference were observed in all the treated groups except MPTP only when compared with the control group in terms of the latency of falls.

\section{Parallel Bar Test}

In this test a significant increase or decrease in latency of turning (LOT) scores were considered as abnormal motor coordination when the treatment groups were compared against control. Although there was no significant LOT in any of the group except the MPTP only group when compared with the control group (NS), there was significance in the time needed to reach edge.

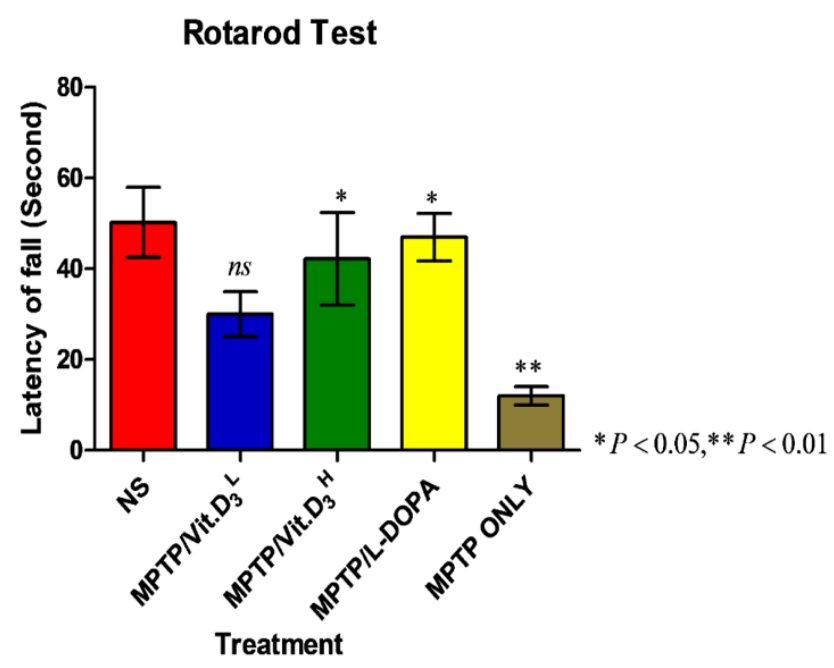

Figure 2: Bar chart showing the latency of fall of the experimental animals in Rotarod Test. The bar chart below represents the passive rotation recorded in rotarod test (Figure 3) for motor function in the mice of the treated group as compared with the control group. No statistically significant difference was observed in all the treated group except MPTP only group when compared with the control group in terms of number of passive rotation recorded in the rotarod test. MPTP induced PD by causing a decline in motor function as seen in MPTP only group ( $\left.{ }^{*} p<0.05\right)$. 


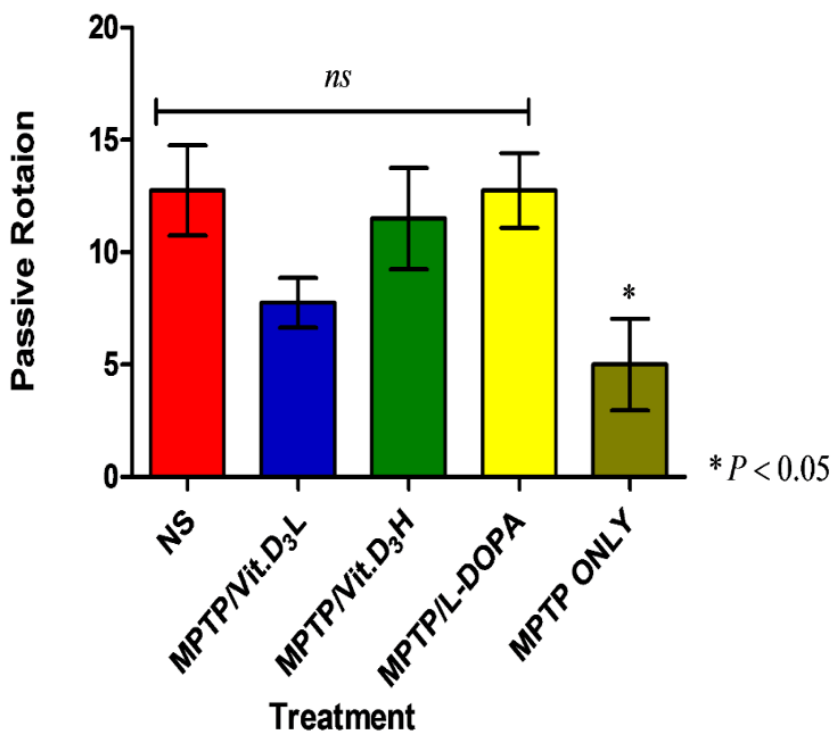

Figure 3: Bar chart showing the Passive rotation recorded in Rotarod test for motor function.

The motor coordination was assessed in the treatment and control group in the parallel bar test to determine the latency of turning (LOT) and significant increase was observed in MPTP only group when compared with control and other experimental groups $(* * * p<0.001)$ (Figure 4) which implies increased in the number of time (sec) it will take the animals in the group to turn either left or right for certain period and that implies significant reduction in motor function recorded in MPTP group as compared to others. A slight significant difference was observed in the MPTP/Vit. $D_{3} L$ when compared with the control group $(* * p<0.01)$ and no statistically significant differences were seen when comparing MPTP/Vit. $\mathrm{D}_{3} \mathrm{H}$, MPTP/L-DOPA and control group which indicate that Vitamin $D_{3}$ at high dosage greatly help to increase motor function better than the low dosage when compared to the control group.

\section{Biochemical assay}

Markers of oxidative stress such as monoamine oxidases, superoxide dismutase, catalase and lipid peroxidation were quantitatively analysed, and the results of the control group were being compared with all the treated groups using one-way ANOVA and post hoc comparison test. The results were graphically represented using bar chart and level of significant were given as $\mathrm{p}<0.05$. From the graph below for Monoamine oxidase assay (Figure 5) using One-way ANOVA and Turkeys Multiple Comparison test, no statistically significant difference was observed when the Vit. $\mathrm{D}_{3} \mathrm{H}$ and L-DOPA groups were compared with control group. However, a very statistically significant difference can be seen in the MPTP/Vit. $D_{3} \mathrm{~L}$ and MPTP treated group in terms of increase level of monoamine oxidase activities when compared with the control group $(* * * p<0.001)$. 


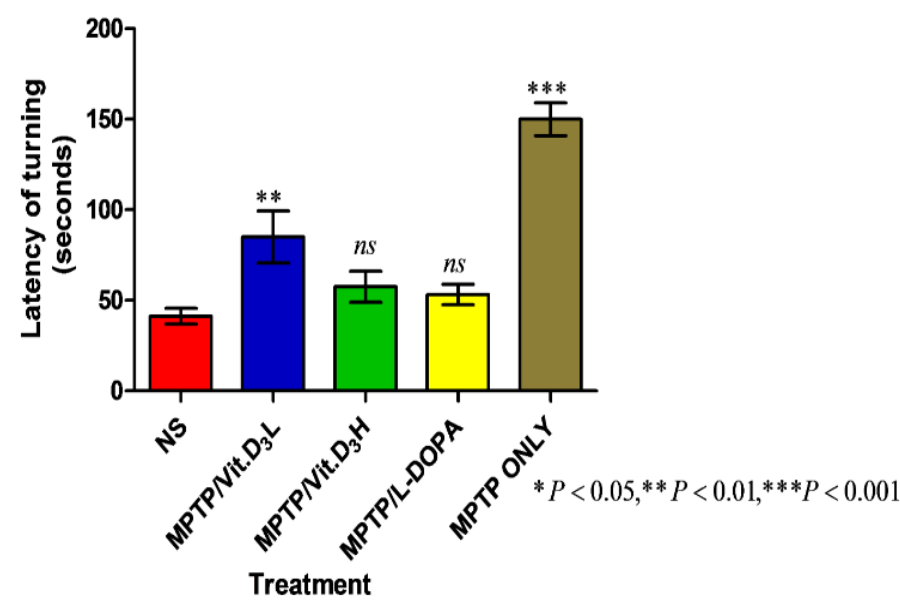

Figure 4: Motor coordination was assessed in the treatment and control group in the parallel bar test to determine the latency of turning (LOT).

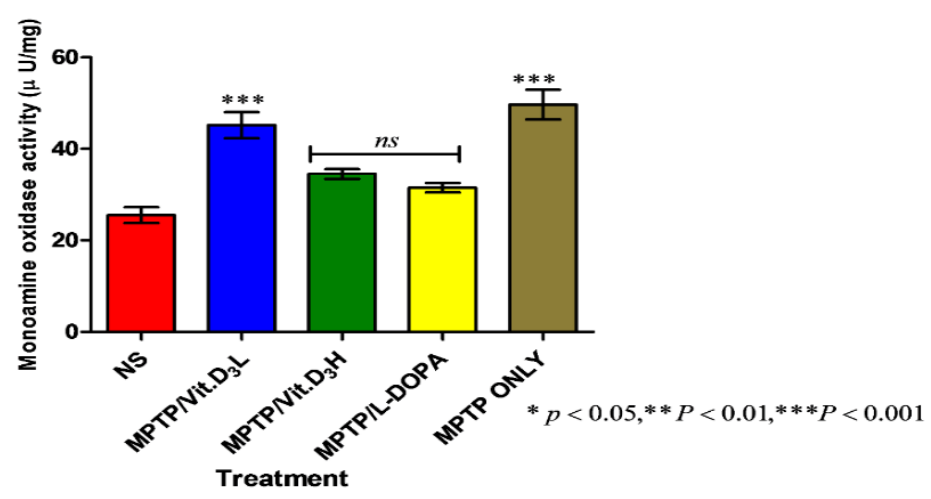

Figure 5: Graph showing the level of Monoamine oxidase activity in experimental animals.

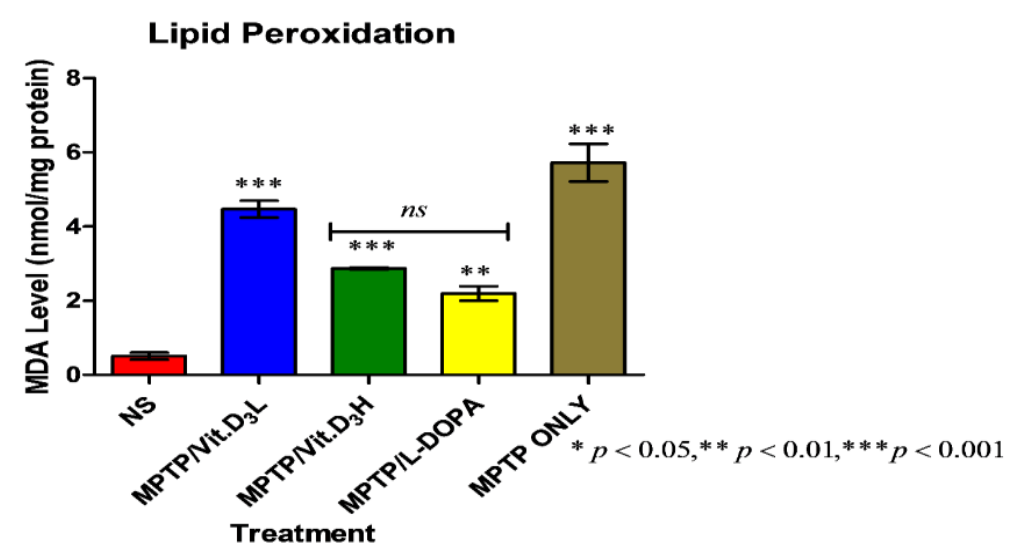

Figure 6: Graph representing the concentration of MDA (Units) per mg in brain tissue.

Figure 6 above showed a slight significant difference in malondialdehyde (MDA) level per mg protein when comparing control group with the MPTP/L-DOPA $(* * p<0.01)$. Also, a statistically significant difference was seen when the MPTP/Vit. $D_{3} \mathrm{H}, M P T P / V i t . D_{3}{ }^{L}$ and MPTP treated group was compared with the control group $(* * * p<0.001)$. No significant difference was seen when compared with the MPTP/Vit. $\mathrm{D}_{3} \mathrm{H}$ and MPTP/L-DOPA group.
From the figure below (Figure 7), there was a statistical significant difference in the catalase activities level in terms of increase which is a marker of oxidative stress in MPTP/Vit. $D_{3} L$ group and MPTP group only when when compared with control group ( $* * * \mathrm{P}<0.001)$. Also, no statistically significant difference was observed when control group was compared with the MPTP/Vit. $\mathrm{D}_{3} \mathrm{H}$ and MPTP/L-DOPA Group 


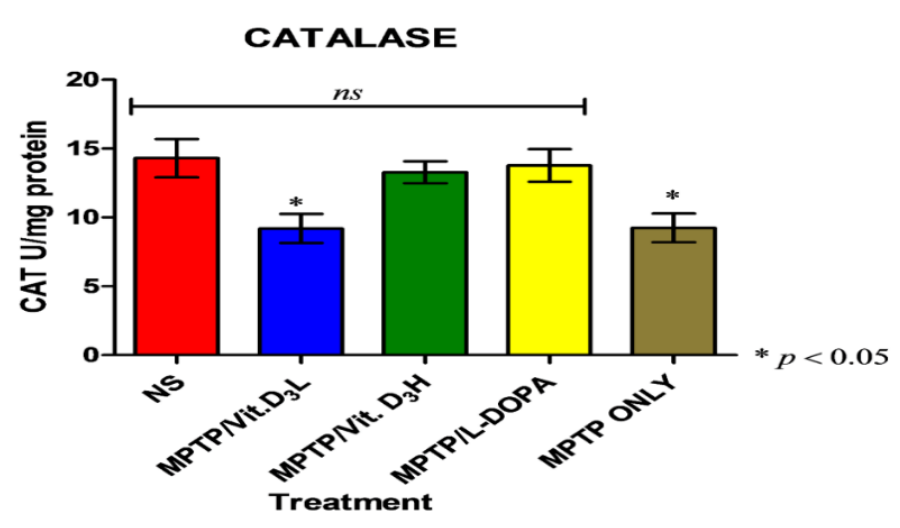

Figure 7: Bar chart representing the concentration of CAT (units) per $\mathrm{mg}$ in brain tissue homogenate.

Figure 8 showed no statistically significant difference in the percentage level of superoxide dismutase activities (SOD) when the control group (NS) was compared with MPTP/Vit. ${ }_{3} \mathrm{H}$ and MPTP/L-DOPA but a
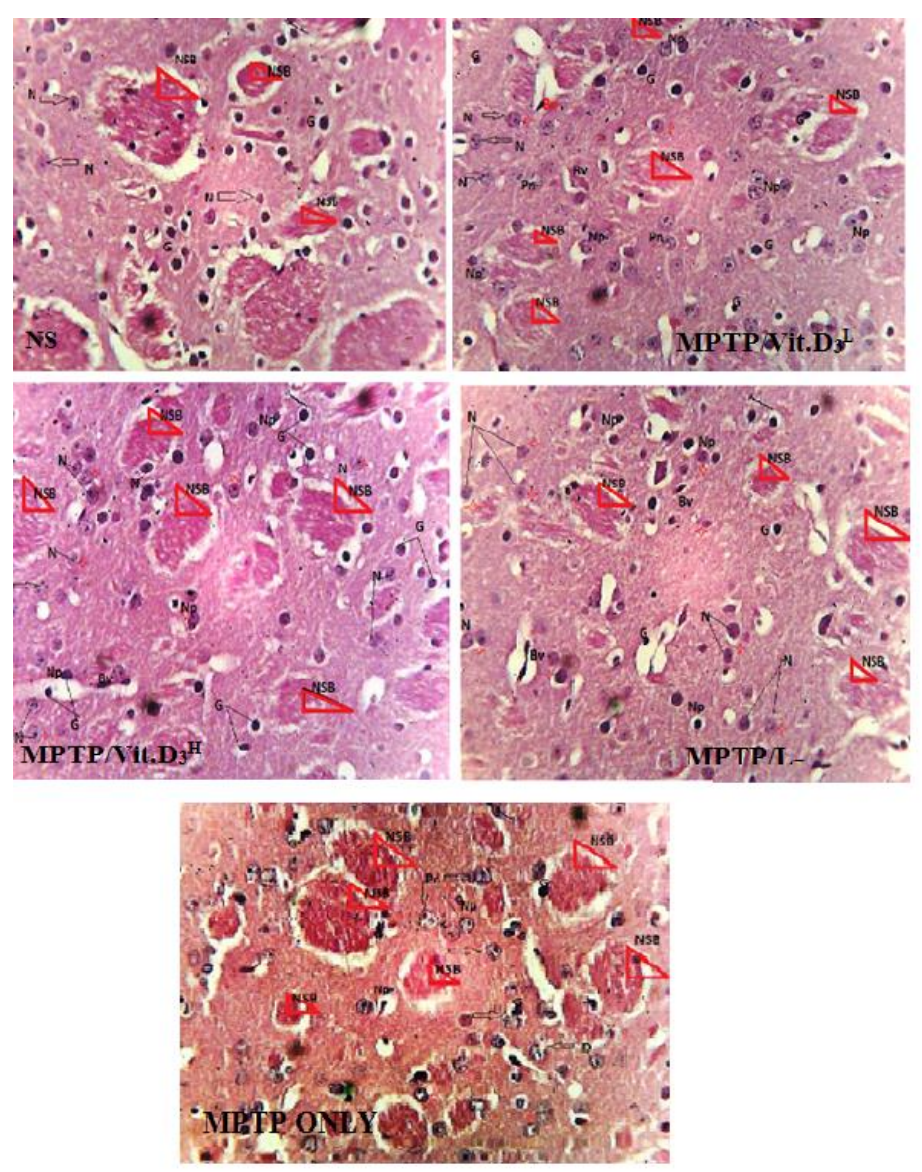

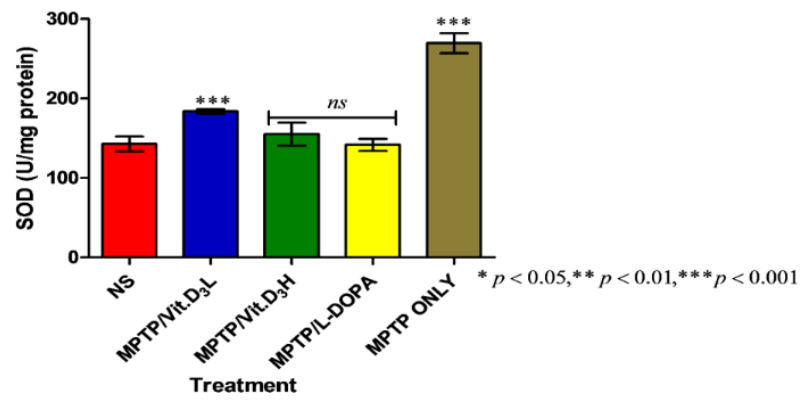

Figure 8: Bar chart showing percentage change of SOD in brain tissue homogenate.

statistical significant increase was observed in the level of SOD of MPTP group only and MPTP/Vit. $D_{3} L$ group when compared with the control group and other experimental groups $\left({ }^{*} p<0.001\right)$.

Figure 9: Photomicrograph of sections of a Striatum (CPu) H\&E. Mgx400. Np: neuropil, NSB: Nigrostriatal bundle fibres (edge arrow), K: Karyorrhexis, Pn: Pyknotic nuclei, pointed arrow - Healthy neuron, Pointed arrow Degenerated neuron

G: Glia cells 

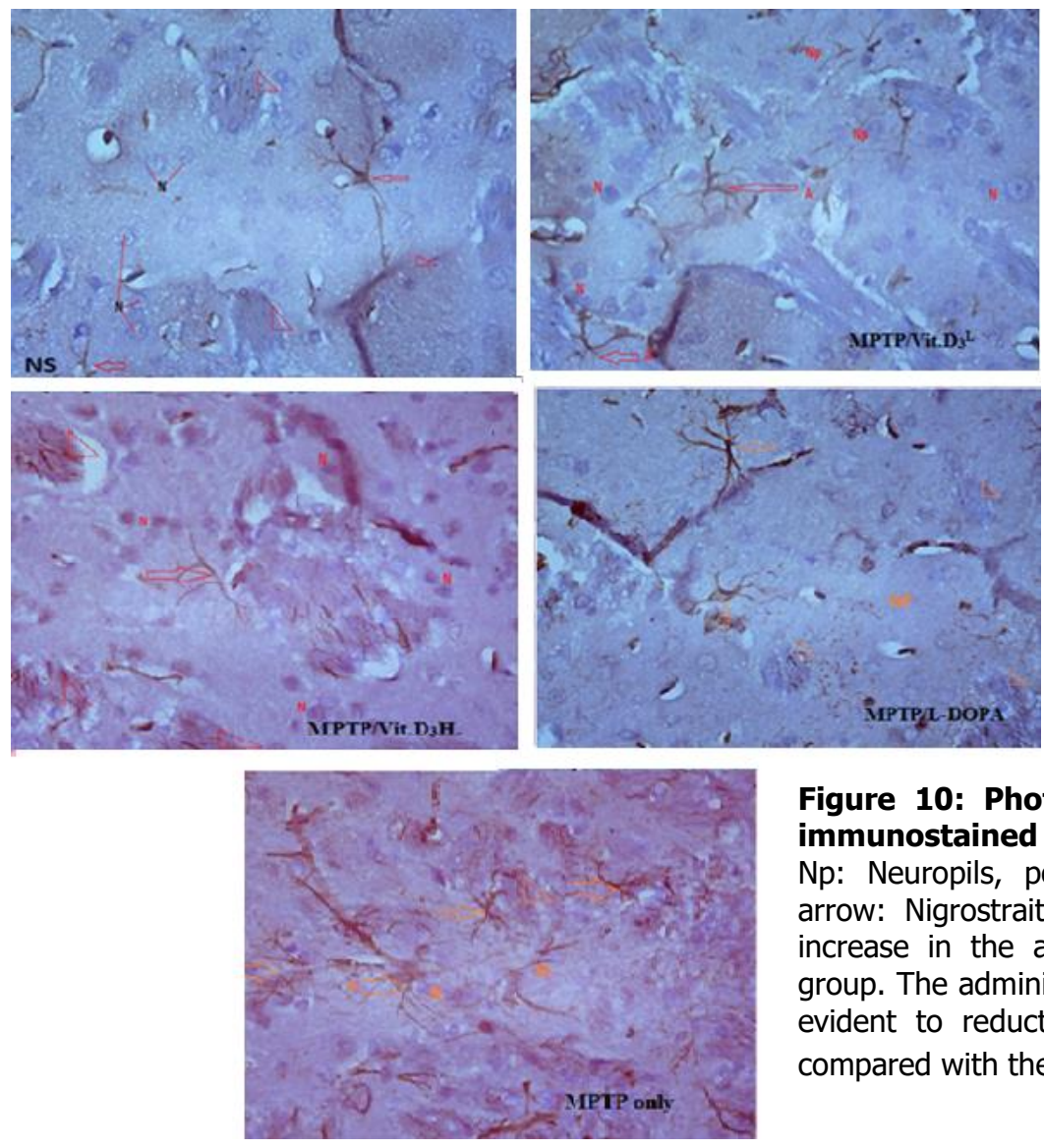

Figure 10: Photomicrograph of $\mathrm{CPu}$ sections immunostained for GFAP Mgx400. N: Neuron, Np: Neuropils, pointed arrow/A: Astrocytes, Edge arrow: Nigrostraital bundles. There is a significant increase in the astrocytic processes in MPTP only group. The administration of Vit. $D_{3}$ in some group is evident to reduction of activated astrocytes when compared with the control and MPTP/L-DOPA group.

Figure 11: Photomicrograph of SNc sections immunostained for GFAP Mgx400. N: Neuron, Np: Neuropils, pointed arrow/A: Astrocytes. There is a pronounced increase in the number of activated astrocytes in MPTP only group. The administration of Vit. $D_{3}$ both at high and low dosage showed evident reduction of activated astrocytic and neuropils when compared with the control and MPTP/L-DOPA group.
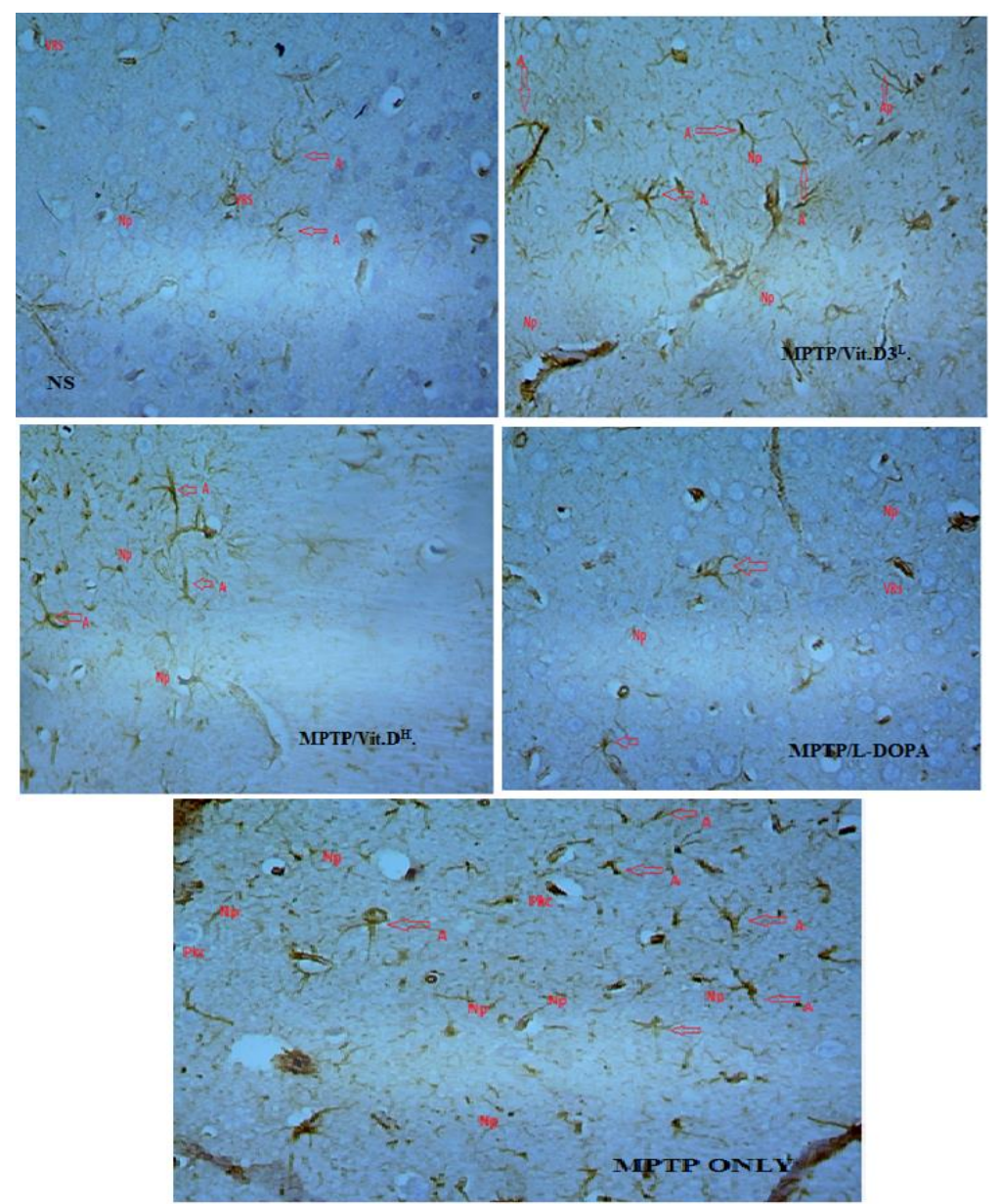
Immunohistochemical studies of section of Striatum (CPu) and Substantia nigra (SNc) from the figure 4-5 revealed the neuroinflammatory effects of MPTP and ameliorative roles of vitamin $D_{3}$ on the glial cells. Increased expression of activated astrocytes seen in MPTP only group is an indication of exposure of neurons in the brain to toxic substances or oxides such as the MPTP which may cause neurodegeneration and motor dysfunction but astrogliosis was reduced significantly in the group treated with Vit. $D_{3}$ at high dose $(100 \mathrm{mg} / \mathrm{kg} \mathrm{BW})$ as compared with the normal control and L-DOPA treated groups $(* \mathrm{p}<0.05, * * \mathrm{p}<0.01, * * * \mathrm{p}<0.001)$.

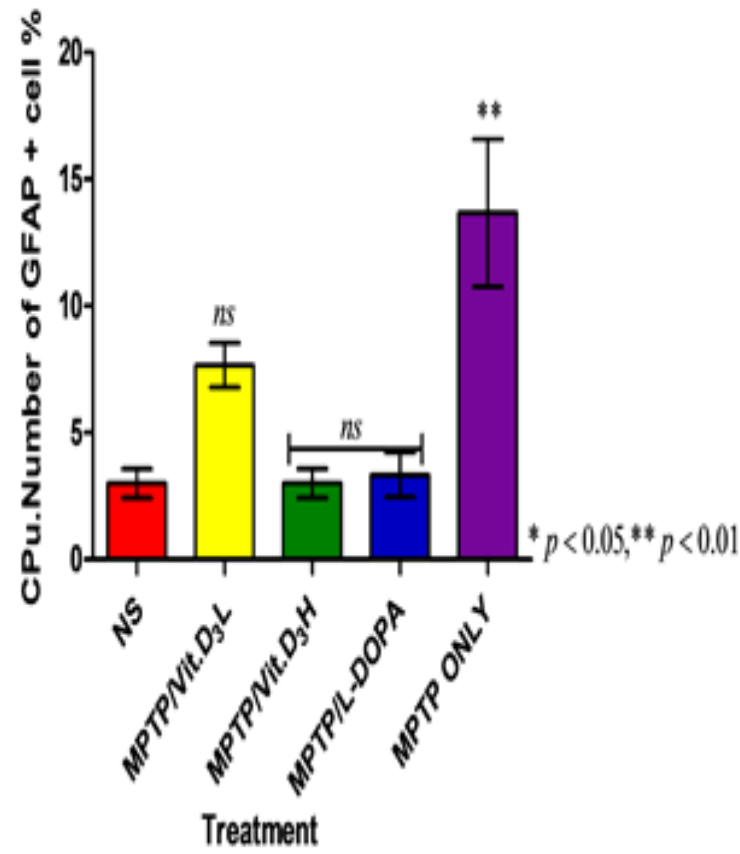

Fig 12: Bar chart showing the Striatal Glial/neurons ratio and number of Striatal GFAP-positive cells.
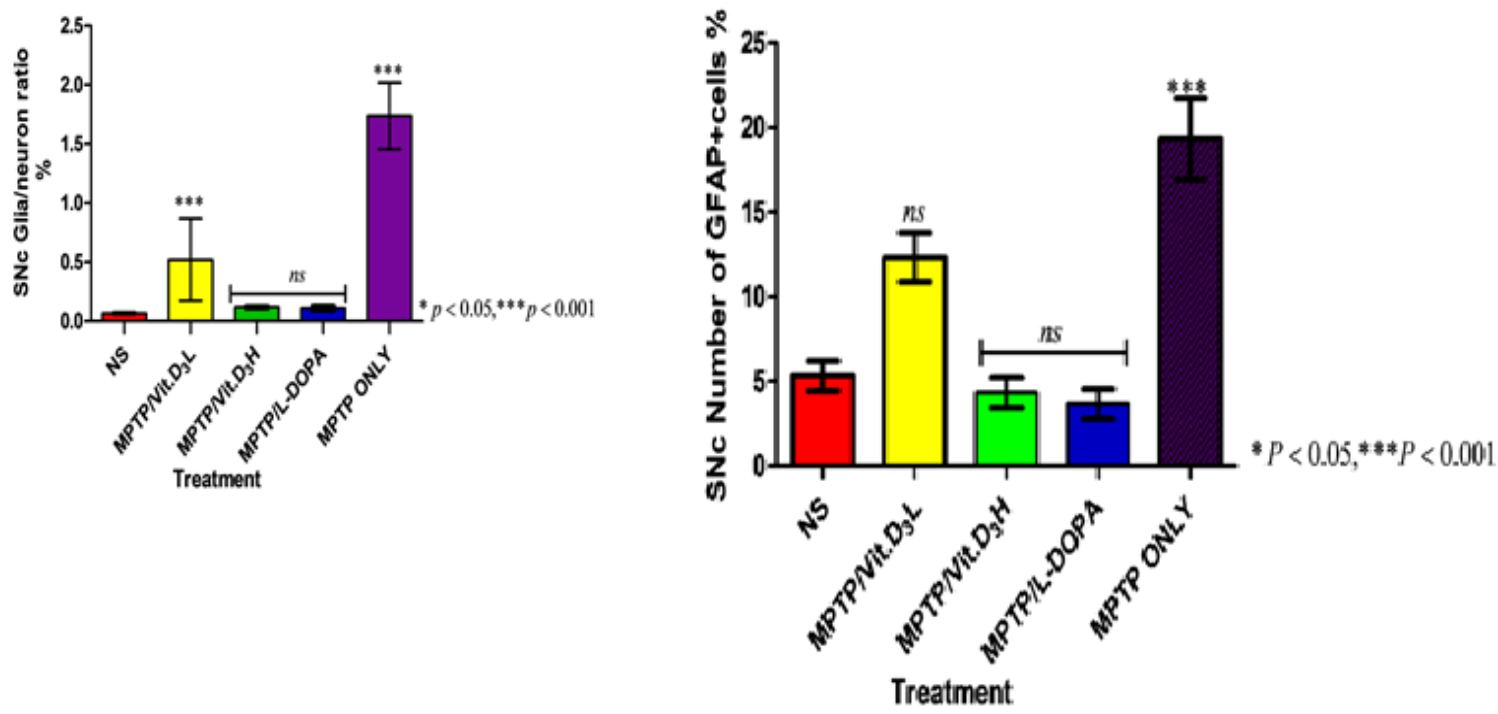

Fig 13: Graphs showing the SNc Glial/neurons ratio and SNc GFAP-positive cells 


\section{DISCUSSION}

This study was designed to test the impact of cholecalciferol (Vit. $D_{3}$ ) stimulation on motor behaviour and immunohistochemical changes in the striatum and substantia nigra after administration of 1-methyl-4-phenyl-1,2,3,6tetrahydropyridine (MPTP) and cholecalciferol $\left(\right.$ Vit. $\left.D_{3}\right)$. Parkinsonism as a symptom is a hallmark of Parkinson's disease and this disease can be induced by selective neurotoxin called MPTP because of its integrating effects and its ability to inhibit complex I activity in mitochondria at the nigrostriatal nerve terminals thereby interfering with adenosine triphosphate (ATP) synthesis which results in augmented production of superoxide anion radicals which is in agreement with Michael and Robert, (2002) and Bazzu et al., (2010). The inhibition is followed by progressive neuroinflammation, neurodegeneration and changes in number of the activated astrocytes in the brain and significant loss of dopamine caused loss of motor function and COordination in mice that received MPTP. Therefore, it is evident from this study that MPTP- induced PD gives rise to degenerative changes in the CPu and SNc which involves loss of neuronal cells and well-marked expression of astrogliosis with impaired motor function which was in line with research done by Langston et al., (1984) and Sai et al., (2013). Furthermore, it was observed that post treatment with Vitamin $D_{3}$ and L-DOPA relieved the toxic effects of the chemical induced PD. This was seen as there was improved motor function in mice that received Vit. $D_{3}$ and LDOPA compared to the group of mice that were given MPTP only for the two weeks of administration. In addition, the Vitamin D therapy seemed to be more effective like that of L-DOPA as demonstrated in the behavioural studies which were further supported by the immunohistochemical analysis where expression of activated astrocytes was minimally reduced in the group of mice that received Vit. $D_{3}$ at high dosage when compared with the control and MPTP/L-DOPA group and this results was in agreement with Wang et al., (2001), Liu et al., (2013), Ogundele et al., (2014); Ishola et al., (2015). Also, there was a statistically increase in the expression of number of GFAP positive cells and percentage $\mathrm{glia} /$ neuron ratio of section in both striatum and substantia nigra with the group that received MPTP only which proves its neurotoxic effect as seen in depletion of dopaminergic neurons and increased activated astrocytes (increased astrogliosis) as supported by Frau et al., (2012), Costa et al., 2013 and James and Asuni, (2013). Four neurobehavioural studies were carried out to assess motor function and co-ordination in mice during the study. The Open field test (OFT) which was done to test exploratory motor activity from figure 1 showed that there was a statistically significant difference when compared the total locomotive activities (TLA) of mice in control group (NS) with the MPTP only and MPTP/VitD ${ }_{3}{ }^{\mathrm{H}}$ groups $(* * * p<0.001)$. It can also be seen that slight statistical significance difference was observed when comparing control group (NS) with MPTP/Vit. $D_{3}{ }^{L}$ group. The rotarod test also showed a statistical significance difference when the control group was compared with the MPTP/Vit. $\mathrm{D}_{3}{ }^{\mathrm{H}}$ group, MPTP/L-DOPA and MPTP only group $\left({ }^{*} p<0.05, * * p<0.01\right)$. With this test, animals with good motor coordination are expected to spend more time on the rotating bar and therefore have a high LOF. No statistical significance was observed when vitamin $D_{3}$ were administered at low dosage (MPTP/Vit. $\mathrm{D}_{3}{ }^{\mathrm{L}}$ ) with the control group (NS) and MPTP/L-DOPA. The result from the rotarod test implies that the role of MPTP in motor dysfunction is linked to its effect on the nigrostriatal dopaminergic pathway where it causes DA depletion in the brain (Burns et al., 1983; Langston et al., 1984; Bankole et al., 2015). There were no significant differences in all the groups when compared with the control group (NS) except with the group that received MPTP only as seen in the passive rotation recorded in rotarod test for motor function. This implies that MPTP may practically induce Parkinson disease which cause a decline in motor function as seen in the group that received MPTP only. Parallel bar test is another neurobehavioural test used to assess motor coordination in experimental mice through the latency of turning (LOT) as seen figure 4. There was only a slight statistical significant 
difference in the latency of turning when comparing the control group (NS) to MPTP/LDOPA group. Also, there was a reduction in motor function in group $E$ which may be as a result of treatment with MPTP only as noticed in the mice with increase time spent to turn from one side to the other when compared with the control and other experimental groups $(* p<0.05, * * p<0.01, * * * p<0.001)$. There was also a statistically significant difference when comparing MPTP/Vit. ${ }_{3}{ }^{H}$ to MPTP/Vit. $D_{3}{ }^{L}$ and MPTP only group. Vitamin $D_{3}$ intervention shows improvement in motor coordination at high dose $(100 \mathrm{mg} / \mathrm{kg})$ as it has also been shown to improve motor deficit in another model of Parkinsonism (Ogundele et al., 2014; Ishola et al., 2015; Bankole et al., 2015). PD is the most common cause of parkinsonism, accounting for $\sim 80 \%$ of cases and the pathological hallmarks of PD are the loss of the nigrostriatal dopaminergic neurons as well as the presence of intraneuronal proteinacious cytoplasmic inclusions, termed "Lewy Bodies" (LBs) (Dauer and Przedborski, 2003). MPTP is said to induce PD by selectively destroying first SNpc neurons that consequently leads to striatal DA deficiency, which is responsible for the major symptoms of PD such as behavioural deficit (Dauer and Przedborski, 2003; Meredith and Redemacher, 2011; Blesa et al., 2012). It was observed from this study that animals given MPTP has low motor coordination, evidence shows reduced LOF and LOT and this is similar to what has been reported that MPTP leads to loss of dopaminergic neurons leading to motor impairment (Dauer and Przedborski, 2003). Animals post treated with Vit. $D_{3}$ showed improvement in motor coordination as they stayed longer on the rotating bar. VDR activation is said to improve motor and cognitive decline in other models of PD (Eyles et al., 2014; Durk et al., 2014; Ogundele and Sanya, 2014; Brown et al., 2003). It is hypothesized that VDR activation helps in alternative activation of DA neurons by calcium signaling. MPTP induced PD caused a decline in motor function when compared with the control. MPTP, when administered into the body crosses the blood brain barriers (BBB) and is converted into activated MPP+ by the actions of MAO-B and other oxidative processes. These trigger the formation of reactive oxygen species (Tatton et al., 1997) which accumulate inside the mitochondria impairing the mitochondrial respiration and indirectly leading to inhibition of complex I thereby initiating cell death-related signaling pathways. L-Dopa which is a conventional drug for PD also shows improvement in motor coordination as it produces all supply of DA in the brain. There was a significant increase in the LOF in rotarod used for motor test on the mice treated with high dose Vit. $D_{3}$. This shows that the high dose Vit. $D_{3}$ treatment which was safe for consumption could leads to the maintenance in motor coordination of the mice; there was also significant increase in LOF for mice treated with L-Dopa. This inability of dopamine to activate $D_{2}$ receptors directly leads to the lack of modulation of motor symptoms seen in Parkinson disease patients (Ogundele et al., 2014). Subsequent Vit. $D_{3}$ in high dose is seen to relieve motor impairments caused by neurotoxin called MPTP after a short period of time. There was a significant decrease in time needed to turn for mice treated with low and high dose Vit. $D_{3}$ when compared with MPTP only in parallel bar test. Reduction of monoamine oxidase $B$ (MAO-B) activity and lipid peroxidation may provide protection against oxidative neurodegeneration caused by MPTP treatment. Monoamine oxidase is actively involved in mapping up monoamines from the synapse. Monoamine activities are not significant when MPTP/Vit. $\mathrm{D}_{3} \mathrm{H}$ and MPTP/L-DOPA groups were compared with control group, but increased level of monoamine activities observed were statistically significant when MPTP/Vit. $D_{3} L$ and MPTP only group were therefore compared with the control group $(* * * p<0.001)$. Since the control animals do not have hyperactivation of the monoamine system, MAO activity will not be high. Animals treated with MPTP should have lost dopaminergic stimulation thereby reducing the amount of dopamine in the brain. Since DA is already low in animals treated with MPTP, MAO activity should also be reduced. Intervention with Vit. $D_{3}$ at high dose after MPTP administration did not show significant increase or reduction in MAO activity; this may be due to the fact that vitamin $D$ receptor is involved in $\mathrm{Ca}^{2+}$ signalling in the brain and not DA synthesis 
(James and Asuni, 2013; Groves et al., 2014). MAO-B activity also showed some slight statistically significant increase when the control group values were analysed using oneway ANOVA and with MPTP/Vit. $\mathrm{D}_{3}{ }^{\mathrm{L}}$ and MPTP only groups. As a result of its role in oxidative stress, MPTP facilitated the increase in lipid peroxidation (MDA) and in due course, Vit. $\mathrm{D}_{3}$ intervention helped in the reduction of lipid peroxidation production caused by MPTP treatment. This was seen by an increased significant difference in MDA level when the control group (NS) was compared with the MPTP/Vit. $\mathrm{D}_{3}{ }^{\mathrm{H}}$ and MPTP/Vit. $\mathrm{D}_{3} \mathrm{~L}$ group $(* * * p<0.001)$. Also, the use of L-DOPA as a drug therapy for the MPTP/L-DOPA group showed a statistical significance difference when compared with the control group $\left(*^{*} \mathrm{p}<0.01\right)$. In summary, it can be inferred that the use of Vit. $D_{3}$ and L-DOPA can reduce oxidative stress and toxicity caused by MPTP in vivo but a statistical significant increase observed in catalase activities in group with MPTP/Vit. $\mathrm{D}_{3}{ }^{\mathrm{L}}$ and MPTP only $(* * * \mathrm{p}<0.001)$. The study revealed that Vit. $\mathrm{D}_{3}{ }^{\mathrm{H}}$ significantly reduced the level of catalase activity in the brain. As a result of the reduction in the level of catalase activity in the brain of the treated animals (MPTP/Vit. $\mathrm{D}_{3} \mathrm{H}$ and MPTP/L-DOPA), this could lead to a significant reduction in oxidative stress which could protect dopaminergic neurons and activated astrocytes, and this was in agreement with Brioukhanov et al., (2006). Increase in the free radicals in the brain has been reported to result in the decrease in density of dopaminergic receptors in the brain and this will result in having low dopamine in the system for longer period of time which in turn result in cell death and glial activation in the dopamine neurons (Michael and Robert, 2002; Bazzu et al., 2010). Furthermore, superoxide dismutase activity (SOD) was represented and the percentage change of SOD in brain tissue homogenate revealed no statistically significant difference when control group was compared with MPTP/Vit. ${ }_{3}{ }^{H}$ MPTP/L-DOPA. However, a statistically significant difference in the SOD activities was observed when control group (NS) was compared with MPTP/Vit. $D_{3}$ and MPTP group in terms of increase of superoxide dismutase level which are signs of oxidative stress $(* * * p<0.001)$. In addition to the results, $\mathrm{H} \& \mathrm{E}$ stains demonstrate general histoarchitectural design of the neurons and this helped to check for structural changes in the striatum (CPu) as well as substantia nigra (SN). Mild neural cell death with appearance of pyknotic nuclei, nuclear fragmentation and increase gliosis was observed with the MPTP treatment groups. From these observations, as stated earlier in this study, it can be said that MPTP caused neurodegeneration which led to loss of dopaminergic neurons in the CPu while the group that received Vitamin $D_{3}$ at high dosage showed an improved presence of neurons, reduced gliosis and preserved nigrostriatal bundles. This means that the Vit. $D_{3}$ treatment has a neuroprotective effect on the motor function by increasing the number of dopaminergic neurons and reducing glia activation.

In conclusion the role of Cholecalciferol (Vit. $\left.D_{3}\right)$ and L-DOPA in drug development can be seen in this study as both drugs helped to improve motor function following MPTP induced PD. This was further supported when Vit. $D_{3}$ intervention helped in the reduction of MDA production caused by MPTP treatment and significantly reduced oxidative stress at a high dose $\left(100 \mathrm{mg} / \mathrm{kg} \mathrm{BW}\right.$ Vit. $\left.D_{3}\right)$. Thus, reduction of lipid peroxidation production by stimulation of vitamin $D$ receptors present in the brain with Vit. $D_{3}$ will reduce oxidative stress, gliosis and neuroinflammation caused by MPTP. It could be inferred that Vit. $D_{3}$ which is dose dependent has a potent antioxidant effect to reduce the intensity and expression of the activated astrocytes (GFAP) that can lead to neuroinflammation.

\section{ACKNOWLEDGEMENTS}

The authors gratefully acknowledge International Brain Research organization/African Regional Committee Bursary (IBRO/ARC Bursary) as well as International Society of Neurochemistry (ISN/CAEN) for their travel grant. Also wish to thank Mr Ekundina Victor, Medical Laboratory Science Afe Babalola University for his invaluable assistance rendered in this research. We also acknowledge 
the help of my Host, Prof Richard Brown, Department of Psychology and Neuroscience, Dalhousie University, Halifax, Canada for neurobehavioural test procedures and apparatus.

\section{Conflict of interest}

The authors declared no conflict of interest.

\section{REFERENCES}

1. Akinyemi RO. 2012. Epidemiology of Parkinsonism and Parkinson's disease in Sub-Saharan Africa: Nigeria profile. Journal of Neurosciences in Rural Practice. 3(3):233-234

2. Bankole OO, Laoye BJ, Sirjao MU, Ishola AO, Oyeleke ED, Balogun WG, Abdulbasit A, Cobham $A E$, Akinrinade ID, Ogundele OM. 2015. Vitamin D3 receptor activation rescued corticostriatal neural activity and improved motor function in -D2R tardive dyskinesia mice model. $J$ Biomedical Science and Engineering. 8:520-530.

3. Bazzu G, Calia G, Puggioni G, Spissu Y, Rocchitta G, Debetto P, Grigoletto J, Zusso M, Migheli R, Serra PA, Desole MS, Miele E. 2010. Alpha-Synuclein- and MPTP-generated rodent models of Parkinson's disease and the study of extracellular striatal dopamine dynamics: a microdialysis approach. CNS Neurol Disord Drug. 9(4):482-90.

4. Blesa J, Phani S, Jackson-Lewis V, Przedborski S. 2012. Classic and New Animal Models of Parkinson's Disease. Journal of Biomedicine and Biotechnology Volume 3

5. Braak H, Braak E. 2000. Pathoanatomy of Parkinson's disease. J Neurol. 247(2):3-10.

6. Bras J, Guerreiro R, Hardy I. 2015. Snapshot: genetics of Parkinson's disease. Cell. 160:570572.

7. Brioukhanov AL, Netrusov AI, Eggen RI. 2006. The catalase and superoxide dismutase genes are transcriptionally up-regulated upon oxidative stress in the strictly anaerobic archaeon methanosarcina barkeri. Journal of Microbiology. 152(6):1671-7.PMID:16735730 DOI: $10.1099 /$ mic. 0.28542-0.

8. Brown RE, Wong AA. 2007. The influence of visual ability on learning and memory performance in 13 strains of mice. J. Learning \& Memory. 14:134-144.

9. Brown J, Bianco JI, McGrath JJ, Eyles DW. 2003. 1,25-dihydroxyvitamin $D_{3}$ induces nervegrowth factor, promotes neurite outgrowth and inhibits mitosis in embryonic rat hippocampal neurons. Neurosci Lett 343: 139-143.

10. Brugnoli A, Napolitano F, Usiello A, Morari M. 2016. Genetic deletion of Rhes or pharmacological blockade of mTORC1 prevent striato-nigral neurons activation in evodopa-induced dyskinesia. Neurobiol Dis. 85:155-63. doi: 10.1016/j.nbd.2015.10.020.

11. Burns, RS, Chiueh CC, Markey SP, Ebert MH, Jacobowitz DM, Kopin IJ. 1983. A primate model of parkinsonism: selective destruction of dopaminergic neurons in the pars compacta of the substantia nigra by N-methyl-4-phenyl-1,2,3,6-tetrahydropyridine. Proc. Natl. Acad. Sci. USA 80, 4546- 4550 .

12. Burkert, R., McGrath, J., Eyles, D. 2003. Vitamin D receptor expression in the embryonic rat brain. Neurosci. Res. Comm. 33: 63-71.

13. Chiu AS, Gehringer MM, Braidy N, Guillemin GJ, Welch JH, Neilan BA. 2012. "Excitotoxic potential of the cyanotoxin $\beta$-methyl-amino-l-alanine (BMAA) in primary human neurons". Toxicon.60(6):1159-1165.doi:10.1016/j.toxicon.2012.07.169.

14. Cicchetti F, Lapointe N, Roberge-Tremblay A, Saint-Pierre M, Jimenez L, Ficke BW, Gross RE. 2005. Systemic exposure to paraquat and maneb models early Parkinson's disease in young adult rats. Neurobiology of Disease 20:360 - 371 .

15. Chung YC, Kim SR, Jin BK. 2010. Paroxetine prevents loss of nigrostriatal dopaminergic

16. neurons by inhibiting brain inflammation and oxidative stress in an experimental model of Parkinson's disease. J Immunol. 185(2):1230-7. doi:10.4049/jimmunol.1000208.

17. Costa G, Frau L, Wardas J, Pinna A, Micaela AP. 2013. MPTP-induced dopamine neurondegeneration and glia activation is potentiated in MDMA pretreated mice. Movement Disorders. 28(14):1957-1965 
18. Darryl WE, Steven S, Robert K, Martin H, John J, McGratha. 2004. Distribution of the Vitamin D receptor and 1a-hydroxylase in human brain. J.Chem Neuro Anat. 29:21-30.

19. Dauer W, Przedborski S. 2003. Parkinson's Disease: Mechanisms and Models. Neuron 39(6):889-909.

20. Dauer W, Kholodilov N, Vila M, Trillat AC, Goodchild R, Larsen KE. 2002. Resistance of alphasynuclein null mice to the Parkinsonian neurotoxin MPTP. Proceedings of the National Academy of Sciences of the United States of America, 99(22):14524-14529.

21. Dawson TM, Dawson VL. 2003. Rare genetic mutations shed light on the pathogenesis of Parkinson's Disease. J. Clin Invest. 111:145-151.

22. Dawson TM, Ko HS, Dawson VI. 2010. Genetic animal models of Parkinson's disease. Neuron. 66:646-661.

23. Dulla YA, Kurauchi Y, Hisatsune A, Seki T, Shudo K, Katsuki H. 2016. Regulatory Mechanisms of Vitamin $D_{3}$ on Production of Nitric Oxide and Pro-inflammatory Cytokines in Microglial BV-2 Cells. Neurochem Res. 41(11):2848-2858.

24. Durk MR, Han K, Chow EC, Ahrens R, Henderson JT, Fraser PE, Pang KS. 2014. 1a,25Dihydroxyvitamin $D_{3}$ reduces cerebral amyloid- $\beta$ accumulation and improves cognition in mouse models of Alzheimers disease. J Neurosci. 34(21):7091-7101.

25. Eyles DW, Liu PY, Josh P, Cui X. 2014. Intracellular distribution of the vitamin D receptor in the brain: comparison with classic target tissues and redistribution with development. Neuroscience. 268:1-9. doi: 10.1016/j.neuroscience.2014.02.042.

26. Frau, L., Simola, N., Plumitallo, A., Morelli, M. 2012. Microglial and astroglial activation by 3,4methylenedioxymethamphetamine (MDMA) in mice depends on $S(+)$ enantiomer and is associated with an increase in body temperature and motility. J. Neurochem. 124: 69-78.

27. Groves NJ, McGrath JJ, Burne TH. 2014. Vitamin D as a neurosteroid affecting the developing and adult Brain. Annual Review of Nutrition. 34:117-141. Doi: 10.1146/annurev-nutr-071813105557.

28. Ishola AO, Laoye BJ, Oyeleke ED, Bankole OO, Sirjao MU, Cobham EA, Balogun WG, Abdulbasit A, Akinrinade ID, Ogundele OM. 2015. Vitamin $D_{3}$ receptor activation rescued corticostriatal neural activity and improved motor cognitive function in -D2R Parkinsonian mice model. $J$ Biomedical science and Engineering. 8:601-615.

29. James LE, Asuni AA. 2013. Parkinson Disease and the Sunshine "Vitamin". J. Disease and Parkinsonism. 3:2-10.

30. Jason L, Eriksen, Wszolek Z, Petrucelli L. 2005. Molecular pathogenesis of Parkinson's disease. Arch.Neurol. 62(3):353-357.

31. Jenner, P. 2003. Oxidative stress in Parkinson's disease. Annals of Neurology 53 (3):26-36.

32. Kim HD, Jeong KH, Jung UJ, Kim SR. 2016. Myricitrin Ameliorates 6-HydroxydopamineInduced Dopaminergic Neuronal Loss in the Substantia Nigra of Mouse Brain. $J$ Med Food. 19(4):374-82. doi: 10.1089/jmf.2015.3581.

33. Kingsbury AE, Cooper M, Schapira AH, Foster OJ. 2001. Metabolic enzyme expression in dopaminergic neurons in Parkinson's disease: an in situ hybridization study. Ann Neurol. 50(2):142-9.

34. Langston JW, Forno LS, Rebert CS, Irwin I. 1984. Selective nigral toxicity after systematicadministration of 1-methyl-4-phenyl 1,2,5,6-tetrahydropyridine(MPTP) in the squirrel monkey. Brain Res. 292:390-394.

35. Lieberman A. 2006. Depression in Parkinson's disease-a review. Acta Neurol Scand 113:1-8.

36. Liu Y, Li YW, Tang YL, Liu X, Jiang JH, Li QG, Yuan JY. 2013. Vitamin D: preventive and therapeutic potential in Parkinson's disease. Curr Drug Metab. 14(9):989-993.

37. McGrath J. 2001. Does 'imprinting' with low prenatal vitamin D contribute to the risk of various adult disorders? Med. Hypotheses. 56:367-371. 
38. Meredith GE, Redemacher DJ. 2011. MPTP Mouse Model of Parkinson's Disease: An update. J. Parkinson's Dis. 1(1):19-33.

39. Michael JZ, Robert EB. 2002. Pathophysiology of Parkinson's disease. Neuropsychopharmacol. 123:1781-1789.

40. Ogundele OM, Nanakumo ET, Ishola AO, Obende OM, Enye LA, Balogun WG, Cobham AE, Abdulbasit A. 2014. NMDAR/+VDR pharmacological phenotype as a novel therapeutic target in relieving motor-cognitive impairments in Parkinsonism. Drug ChemToxicol. 4:1-13.

41. Ogundele OM, Sanya OJ. 2014. NMDA/ RVDR in fish melanocytes; Receptor targeted therapeutic model and mechanism in parkinson diseases. J.Biomol Res Ther 3:3.

42. Okubadejo NU, Ojo OO, Oshinaike OO. 2010. Clinical profile of Parkinsonism and Parkinson's disease in Lagos, Southwestern Nigeria. BMC Neurology.10:1.

43. Paxinos G, Franklin KB. 2008. The Mouse Brain in Stereotaxic Coordinates, 3rd edition.Academic Press, San Diego, CA.

44. Podhorna J, Brown RE. 2002. Strain differences in activity and emotionality do not account for differences in learning and memory performance between C57BL and DBA/2 mice. Genes Brain and Behavior. 1:96-110.

45. Poewe W. 2008. Non-motor symptoms in Parkinson's disease. European Journal of Neurology 15: 14 - 20.

46. Sai T, Uchida K, Nakayama H. 2013. Biochemical evaluation of the neurotoxicity of MPTP and $\mathrm{MPP}+$ in embryonic and newborn mice. J Toxicol Sci. 38(3):445-58.

47. Sai T, Uchida K, Nakayama H. 2013. Involvement of monoamine oxidase-B in the acute neurotoxicity of MPTP in embryonic and newborn mice. Exp Toxicol Pathol. 65(4):365-73. doi: $10.1016 /$ j.etp.2011.11.003.

48. Shahed J, Jankovic J. 2007. Motor symptoms in Parkinson's disease. Handbook of Clinical Neurology 83:329-342.

49. Song DD, Shults CW, Sisk A, Rockenstein E, Masliah E. 2004. Enhanced substantia nigra mitochondrial pathology in human alpha-synuclein transgenic mice after treatment with MPTP. Experimental Neurology. 186(2):158-172.

50. Tatton, NA, Kish, SJ. 1997. In situ detection of apoptotic nuclei in the substantia nigra compacta of 1-methyl-4-phenyl-1,2,3,6-tetrahydropyridine-treated mice using terminal deoxynucleotidyl transferase labelling and acridine orange staining. Neuroscience. 77:10371048.

51. Uberti F, Morsanuto V, Bardelli C, Molinari C. 2016. Protective effects of 1a,25Dihydroxyvitamin $\mathrm{D}_{3}$ on cultured neural cells exposed to catalytic iron. Physiol Rep. 4(11): 120-129. doi: $10.14814 /$ phy2.12769.

52. Van Den Eeden SK, Tanner CM, Bernstein AL, Fross RD, Leimpeter A, Bloch DA. 2003. Incidence of Parkinson's disease: Variation by age, gender and race/ethnicity. American Journal of Epidemiology. 157 (11):1015-1022.

53. Wang D, Qian L, Xiong H, Liu J, Neckameyer WS, Oldham S. 2006. Antioxidants protect PINK1 dependent dopaminergic neurons in Drosophila. Proceedings of the National Academy of Sciences of the United States of America, 103(36): 13520-13525.

54. Wang JY, Wu JN, Cherng TL, Hoffer BJ, Chen HH, Borlongan CV, Wang Y. 2001. Vitamin $D_{3}$ attenuates 6-hydroxydopamineinduced neurotoxicity in rats. Brain Res. 904:67-75.

55. Wyrsch P, Blenn C, Bader J, Althaus F. 2012. Cell Death and Autophagy under Oxidative Stress: Roles of Poly(ADP-Ribose) Polymerases and Ca ${ }^{2+}$ Mol. Cell. Biol. 32(17) 3541-3553. 